\title{
AIP
}

\section{Extending the dynamic range of nuclear pulse spectrometers}

\author{
A. Pullia, F. Zocca, G. Pascovici, and D. Bazzacco
}

Citation: Rev. Sci. Instrum. 79, 036105 (2008); doi: 10.1063/1.2894305

View online: http://dx.doi.org/10.1063/1.2894305

View Table of Contents: http://rsi.aip.org/resource/1/RSINAK/v79/i3

Published by the American Institute of Physics.

\section{Related Articles}

Calibration of a Thomson parabola ion spectrometer and Fujifilm imaging plate detectors for protons, deuterons, and alpha particles

Rev. Sci. Instrum. 82, 073301 (2011)

Detection of dust on JET with the high resolution Thomson scattering system

Rev. Sci. Instrum. 81, 10E131 (2010)

Application and methodological improvements to the floating-wire technique to characterize the magnetic properties of a spectrometer dipole

Rev. Sci. Instrum. 72, 2003 (2001)

A time detector design for $\mathrm{MeV}$ particles using secondary electron emission and microchannel plates

Rev. Sci. Instrum. 68, 4027 (1997)

Extrapolation procedures in Mott electron polarimetry

Rev. Sci. Instrum. 63, 114 (1992)

\section{Additional information on Rev. Sci. Instrum.}

Journal Homepage: http://rsi.aip.org

Journal Information: http://rsi.aip.org/about/about_the_journal

Top downloads: http://rsi.aip.org/features/most_downloaded

Information for Authors: http://rsi.aip.org/authors

\section{ADVERTISEMENT}

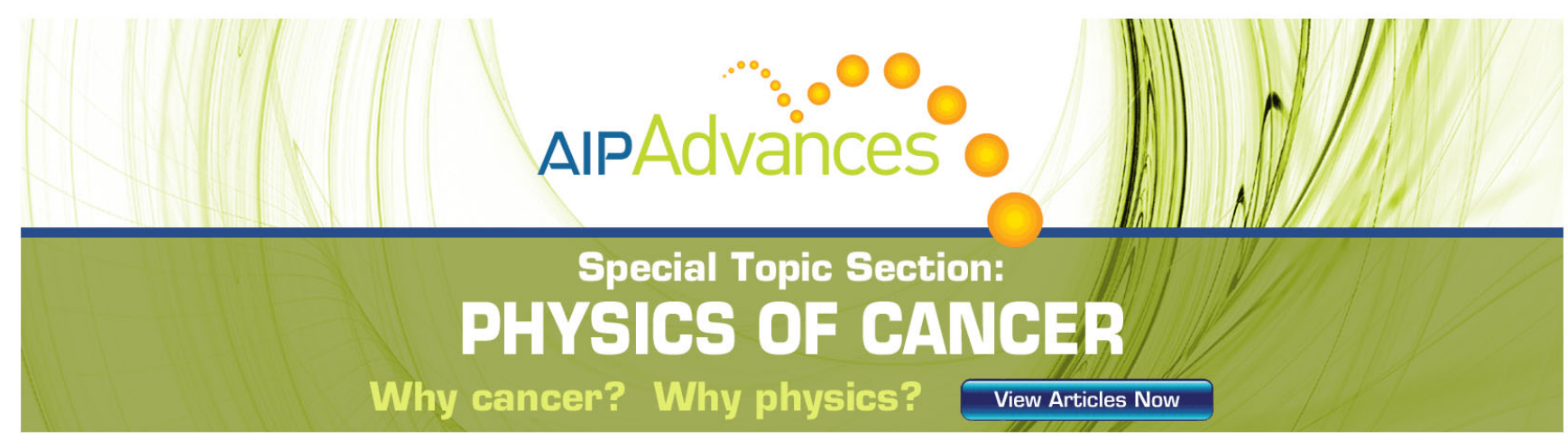




\title{
Extending the dynamic range of nuclear pulse spectrometers
}

\author{
A. Pullia, ${ }^{1,2}$ F. Zocca, ${ }^{1,2}$ G. Pascovici, ${ }^{3}$ and D. Bazzacco ${ }^{4}$ \\ ${ }^{1}$ Department of Physics, University of Milano, via Celoria 16, I-20133 Milano, Italy \\ ${ }^{2}$ INFN Milano, via Celoria 16, I-20133 Milano, Italy \\ ${ }^{3}$ University of Koeln, IKP, Zuelpicher Strasse 77, D-50937 Koeln, Germany \\ ${ }^{4}$ INFN Padova, via Marzolo 8, I-35131 Padova, Germany
}

(Received 18 January 2008; accepted 18 February 2008; published online 17 March 2008)

\begin{abstract}
Using an innovative time-varying front-end electronics in conjunction with a bulky coaxial high-purity germanium detector, we were able to extend the range of the radionuclide spectra well beyond the analog to digital converter (ADC) saturation point. The electronics automatically conditions the signal for digital-filtering optimization if it is in the ADC voltage range and for time-over-threshold analysis if it exceeds the ADC range. A high spectroscopic resolution has been achieved in both operation ranges. An unprecedented wide energy range from $5 \mathrm{keV}$ to $150 \mathrm{MeV}$ of equivalent energy, or $90 \mathrm{~dB}$, has been obtained using a single acquisition chain, while maintaining a high-energy resolution in the whole spectrum. For example, with an ADC range of $3 \mathrm{MeV}$ a resolution has been obtained of $1.3 / 2.2 \mathrm{keV}$ full width at half maximum on the $122 / 1332 \mathrm{keV}$ gamma-ray lines of ${ }^{57} \mathrm{Co}$ and ${ }^{60} \mathrm{Co}$, and of $<0.4 \%$ in the time-over-threshold region, or for energy deposits beyond $3 \mathrm{MeV}$. (C) 2008 American Institute of Physics. [DOI: 10.1063/1.2894305]
\end{abstract}

In this Note we suggest that high-resolution spectroscopy of ionizing radiations ( $\mathrm{X}$ and gamma photons, charged particles, ions) is possible even beyond the ADC range, by using a proper time-varying preamplifier and switching to a time-over-threshold (TOT) measurement technique for the over-range pulses. The typical case in which such an approach can be fully exploited is with thick high-purity semiconductor detectors such as $\mathrm{Ge}, \mathrm{Si}(\mathrm{Li}), \mathrm{CdTe}$, etc., where the measurement range is typically limited by the ADC itself or by the output stages of the analog electronics, rather than by the detector. Normally such cases are addressed by reducing the gain of the electronic chain or by using two or more electronic chains in parallel with different gain values. The former approach typically yields a poor resolution at the lower energies and suffers from noise and disturbances picked up at the circuit output, while the latter requires a complex multichannel acquisition architecture for each individual detector channel. The proposed approach overcomes these issues and provides through a single acquisition channel a high resolution and an excellent noise immunity over the full dynamic range of the detector. The technique is demonstrated using a HPGe capsule of the AGATA detector ${ }^{1}$ and an ${ }^{241} \mathrm{Am}+\mathrm{Be}$ source with $\mathrm{Ni} / \mathrm{Fe}$ target setups. ${ }^{2}$

In Fig. 1 the structure of the used front-end electronics is shown. It consists of a charge-sensitive preamplifier ac coupled to the detector anode $\left(c_{\mathrm{ac}}\right)$, connected to a passive pole-zero cancellation stage with a fast-reset circuitry, comprising a Schmitt-trigger comparator $(T)$ and a switched constant-current sink $\left(I_{R}\right)$. The higher threshold $\left(T_{H}\right)$ of the comparator is set at or slightly below the ADC maximum input voltage. The lower threshold $\left(T_{L}\right)$ is set at $0 \mathrm{~V}$. A gain/ antialiasing (GAA) stage is used to pass the signal along to a 14 bit $100 \mathrm{MHz}$ flash ADC. The sampled signals are stored into a personal computer hard disk through a universal serial bus interface and processed off line using either standard digital-filtering techniques, ${ }^{3-5}$ or the TOT algorithm de- scribed next, depending on whether their amplitude is under or over $T_{H}$.

The charge-sensitive preamplifier itself has a very large voltage range of $11 \mathrm{~V}$, with a linear range of $8 \mathrm{~V}$ or of more than $150 \mathrm{MeV}$ of equivalent energy. However, after the GAA stages, the ADC can cover only a little fraction of this range or $3 \mathrm{MeV}$ of equivalent energy. Reduction of the circuit gain would remarkably spoil the energy resolution at the lower energies because of second-stage and pickup noises and therefore is not envisaged. A question arises: how to measure the large signals yielding ADC saturation ? Here is the idea: when the signal overcomes the ADC range, switch $S$ is turned on by comparator $T$ and current $I_{R}$ is continuously flown through capacitance $C$ until the output voltage reaches its baseline at $0 \mathrm{~V}$. At that point switch $S$ is automatically turned off by $T$. The time needed to complete this process, or TOT for the shake of brevity, depends on the amplitude of the input signal. So a measurement of the TOT yields a measurement of the signal amplitude. Note that the working principle of this circuit is similar to that of Wilkinson ADCs. ${ }^{6}$ In order to accurately measure the reset time, we sampled the signal provided by the comparator using one more 14 bit $100 \mathrm{MHz}$ ADC channel and interpolated the leading and trailing edges of the sampled waveform so as to derive its width at half amplitude with a subsampling-time resolution. In Fig. 2 one such acquired large signals is shown along with the corresponding comparator signal. We accurately calculated the expression of the reset transient, and we found an exponential relation between the reset time and the total equivalent energy that is subject to reset. This energy is given by the sum of the energy of the large overthreshold event and the energy of the tail due to previous sub-threshold events. By expanding the exponential time-to-energy relation to second order, while also including the tail contribution and an offset term, the following relation is found: 


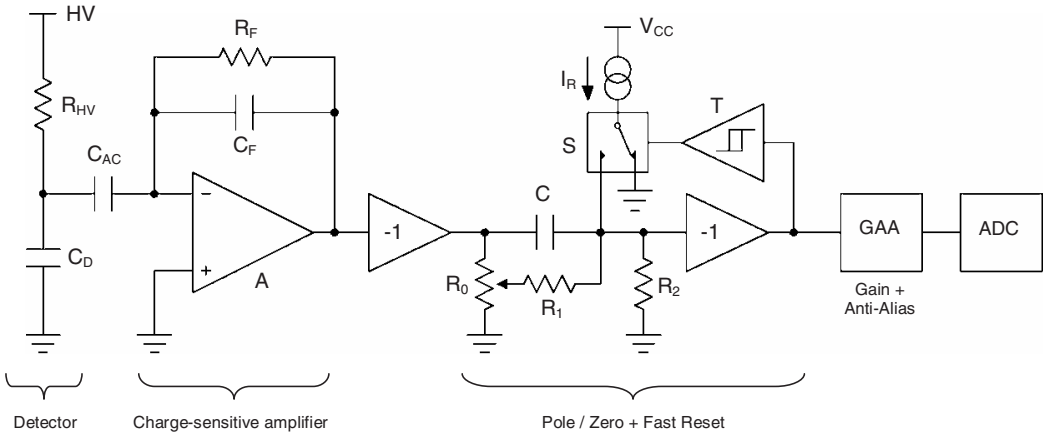

FIG. 1. Structure of the used front-end electronics. HV is the $+4 \mathrm{kV}$ high voltage used for detector biasing and $C_{\mathrm{AC}}$ is the ac coupling capacitor.

$$
E=b_{1} T+b_{2} T^{2}-k_{1}\left(V_{1}-V_{2}\right)+E_{0},
$$

where $E$ is the unknown energy of the event, $T$ is the reset time, and $V_{1}$ and $V_{2}$ are the pre- and postevent baselines. $b_{1}$, $b_{2}, k_{1}$, and $E_{0}$ are constant parameters that can be easily derived once for all through a least-mean-square fitting procedure run on a calibration dataset. The first term on the right hand of Eq. (1) dominates over the second, so parameter $b_{1}$ well represents the "reset velocity" which, in our case, is of $\sim 4.2 \mathrm{MeV} / \mu \mathrm{s}$.

We tested the new measurement technique using an HPGe detector of AGATA (core electrode) as irradiated with an ${ }^{241} \mathrm{Am}+\mathrm{Be}$ source housed in a cylindrical box with thick paraffine walls for fast-neutron thermalization and we used a $\mathrm{Ni}$ powder or Fe target for neutron capture production of gamma photons in the 3-9 $\mathrm{MeV}$ energy range. We also used a few other simpler calibration sources $\left({ }^{57} \mathrm{Co},{ }^{60} \mathrm{Co}\right)$. In Fig. 3 a spectrum is shown of this source as acquired beyond the ADC range with our front-end electronics working in the TOT mode. The energy of the events is derived from the reset time through relation (1). The overall count rate of the events was $\sim 3.5 \mathrm{kHz}$. The part of the spectrum at energies $<3 \mathrm{MeV}$ has been also acquired in normal pulse-height mode, but is not shown here.

For comparison we also show in Fig. 4 the same spectrum as acquired in amplitude mode only, after reducing the

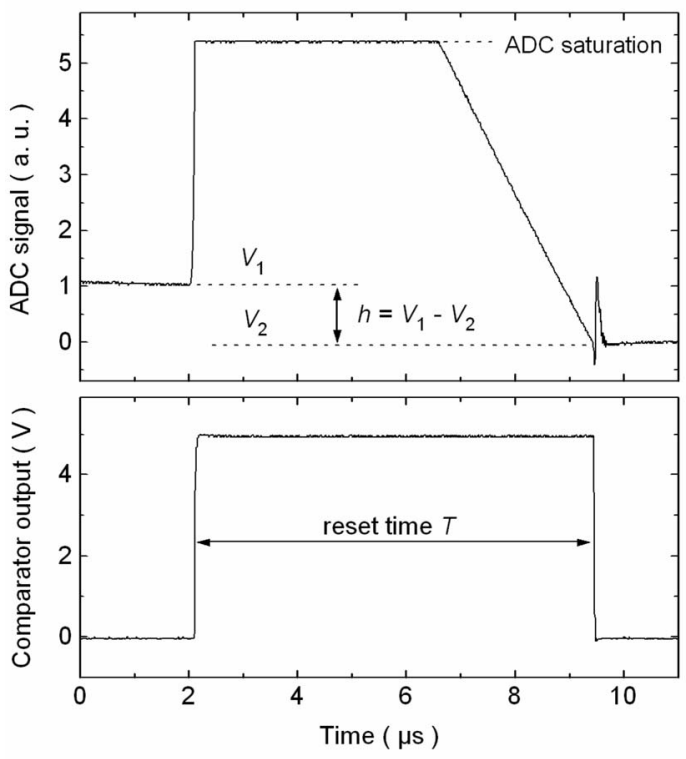

FIG. 2. Top: large signal waveform superposed to the tail of a previous event. The flat top is caused by ADC saturation. Bottom: corresponding signal provided by the comparator. electronic chain gain in such a way that the ADC range reached $9.4 \mathrm{MeV}$. Note that in this case the false line at 9.4 MeV is due to ADC saturation and not to physical events. Note also that gain reduction yielded a worsening of $\sim 0.2 \mathrm{keV}$ full width at half maximum (FWHM) in the energy resolution at low energy, i.e., on the ${ }^{57} \mathrm{Co}$ line, as caused by the higher impact of second stage and pickup noises.

The excellent correspondence of the spectra of Figs. 3 and 4 is evident, which proves the effectiveness of the TOTmode measurement technique.

In Table I the energy resolution obtained with the new setup of Fig. 1 is shown as evaluated on the various spectral lines of the spectrum. It is worth observing that the width of the carbon line at $4.440 \mathrm{MeV}$ region is intrinsically broadened by the Doppler effect. In fact, the line comes from the capture reaction involving the light nuclei of $\mathrm{Be}$ and the $\alpha$

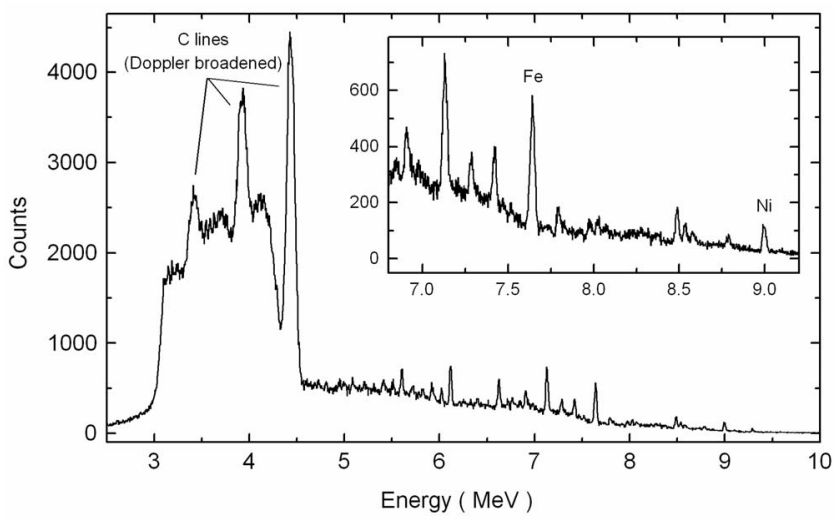

FIG. 3. Spectrum acquired beyond the ADC range of $\sim 3 \mathrm{MeV}$ using the TOT mode technique.

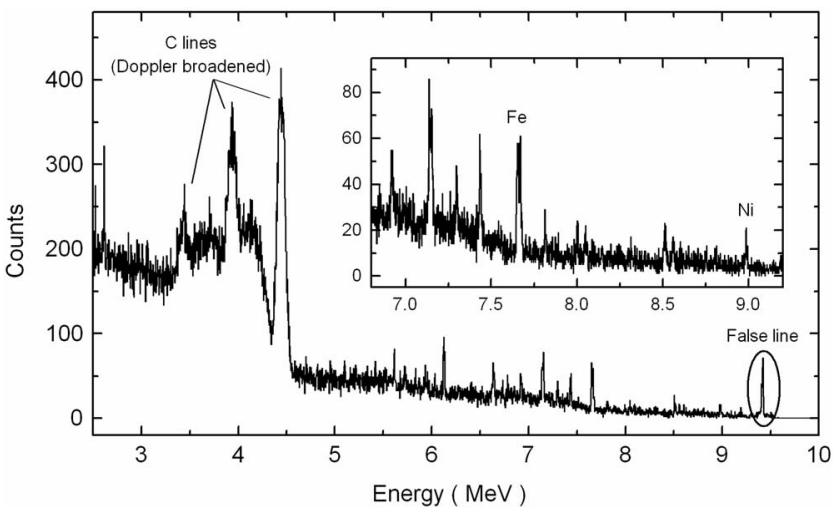

FIG. 4. Spectrum acquired in amplitude mode after reduction of the electronic chain gain. The peak at $9.4 \mathrm{MeV}$ is false and due to ADC saturation. 
TABLE I. Energy resolution.

\begin{tabular}{lcc}
\hline \hline \multicolumn{1}{c}{ Energy } & Resolution FWHM & Mode \\
\hline $122 \mathrm{keV}\left({ }^{57} \mathrm{Co}\right)$ & $1.3 \mathrm{keV}$ & Standard filtering \\
$1.33 \mathrm{MeV}\left({ }^{60} \mathrm{Co}\right)$ & $2.2 \mathrm{keV}$ & Standard filtering \\
$4.440 \mathrm{MeV}(\mathrm{C})$ & $2.34 \%^{\mathrm{a}}$ & TOT \\
$\sim 5.6 \mathrm{MeV}$ & $0.34 \%$ & TOT \\
$\sim 6.1 \mathrm{MeV}$ & $0.28 \%$ & TOT \\
$7.6312 \mathrm{MeV}(\mathrm{Fe})$ & $0.21 \%$ & TOT \\
$7.6456 \mathrm{MeV}(\mathrm{Fe})$ & $0.21 \%$ & TOT \\
$8.9984 \mathrm{MeV}(\mathrm{Ni})$ & $0.21 \%$ & TOT \\
$\sim 19 \mathrm{MeV}$ (pulser) & $0.10 \%$ & TOT \\
$\sim 33 \mathrm{MeV}$ (pulser) & $0.081 \%$ & TOT \\
$\sim 50 \mathrm{MeV}$ (pulser) & $0.086 \%$ & TOT \\
\hline
\end{tabular}

${ }^{\mathrm{a}}$ Dictated mainly by the Doppler effect (see text).

particles emitted by the ${ }^{241} \mathrm{Am}$ source. The resulting ${ }^{12} \mathrm{C}$ nuclei emit $\gamma$ photons while moving after absorption of the $\alpha$ particle momentum. The random angle of the emitting nucleus trajectory yields a remarkable Doppler-effect broadening. All the other lines acquired in the TOT mode show an excellent energy resolution better than $0.4 \%$. Even if the physical line of largest energy is that of $\mathrm{Ni}$ at about $9 \mathrm{MeV}$, we could test the system for equivalent energies $>9 \mathrm{MeV}$ using a high-precision pulser to simulate large detector signals. As can be seen in Table I the pulser lines are in the 19-50 MeV. We had no possibility to inject larger test signals with the pulser available in the used experimental setup. However, in testbench measurements we measured a circuit range of $>150 \mathrm{MeV}$, i.e., an output voltage swing $>8 \mathrm{~V}$ as seen at the charge-sensing stage output.
We believe that this new approach opens the way to all those applications where high-energy deposits (from charged particles, ions, gammas) are to be measured while maintaining at the same time the highest possible energy resolution in the typical lower-energy range.

In conclusion, a new time-variant circuit technique has been proposed for nuclear pulse spectroscopy, which permits a substantial improvement of the energy measurements dynamic range. The technique has been successfully tested using a capsule of the AGATA detector array, an advanced front-end electronics, and an ${ }^{241} \mathrm{Am}+\mathrm{Be}$ neutron source with $\mathrm{Ni} / \mathrm{Fe}$ targets generating high-energy gamma photons by neutron capture reactions. The AGATA preamplifiers fully support the shown technology, which will therefore be available for the next-generation nuclear physics experiments.

The limiting factor of the shown technique is ADC blindness to pileup along the reset transients. Increasing the reset velocity or reducing the gain can help in this regard.

The authors gratefully thank B. Million and A. Bracco for strongly supporting this work and for their valuable suggestions and hints.

${ }^{1}$ D. Bazzacco, B. Cederwall, J. Cresswell, G. Duchene, J. Eberth, W. Gast, J. Gerl, W. Korten, I. Lazarus, R. M. Lieder, J Simpson, D. Weisshaar, http://agata.pd.infn.it/documents/Agata-proposal.pdf.

${ }^{2}$ J. G. Rogers, M. S. Andreaco, C. Moisan, and I. M. Thorson, Nucl. Instrum. Methods Phys. Res. A 413, 249 (1998).

${ }^{3}$ A. Georgiev, W. Gast, and R. M. Lieder, IEEE Trans. Nucl. Sci. 41, 1116 (1994)

${ }^{4}$ A. Pullia, G. Gritti, and G. Ripamonti, IEEE Trans. Nucl. Sci. 44, 331 (1997)

${ }^{5}$ A. Pullia, Nucl. Instrum. Methods Phys. Res. A 397, 414 (1997).

${ }^{6}$ For example, http://www.ortec-online.com/pdf/camactut.pdf. 